\title{
Matrix-assisted laser desorption/ionisation time-of- flight mass spectrometry (MALDI-TOF MS) applied to diatom identification: influence of culturing age
}

\author{
A. Nicolau ${ }^{*, * *}$, L. Sequeira**, C. Santos, M. Mota \\ Institute for Biotechnology and Bioengineering (IBB), Centre of Biological Engineering, University of Minho, \\ Campus de Gualtar, 4710-057 Braga, Portugal
}

\begin{abstract}
Diatom identification is based on classical morphological methods focused on frustule shape and ornamentation analysis and possible chain formation. Because many morphological aspects are common to several species, diatom identification and characterisation are timeconsuming and demand significant expertise. Furthermore, the use of advanced microscopy, such as transmission electron microscopy or scanning electron microscopy, is frequently necessary to differentiate these organisms. Matrix-assisted laser desorption/ionisation time-of-flight mass spectrometry (MALDI-TOF MS) was used to differentiate diatom taxa, and this report presents a preliminary study of the possibility of using this technique to obtain reliable fingerprinting of diatoms in the range of 2 to $20 \mathrm{kDa}$. Seminavis robusta, Coscinodiscus sp., Thalassiosira sp. and Cyclotella meneghiniana were used. The influence of culture age on reproducibility was studied. MALDI-TOF MS spectra were shown to vary with culturing time, and the need to consider this when setting up experimental standard conditions in the identification of diatoms was clearly established. One of the most important observations was that each diatom strain presented a specific age when the mass spectrum became reproducible. For all S. robusta strains and for C. meneghiniana, this age was $9 \mathrm{~d}$, and for Thalassiosira sp. and Coscinodiscus sp., this age was $13 \mathrm{~d}$. Even with the limitations of culture aging versus spectra reproducibility, MALDI-TOF MS proved to be a useful complementary tool to identify and characterise diatoms at the genus level. However, it was not possible to distinguish different mating types within the same species, as evidenced by the results obtained with $S$. robusta isolates.
\end{abstract}

KEY WORDS: Diatoms · MALDI-TOF MS · Culture age

\section{INTRODUCTION}

Diatoms (class Bacillariophyceae) are microscopic photosynthetic organisms (Scala \& Bowler 2001, Gordon et al. 2009) that live in aquatic ecosystems, both marine and freshwater (Gröger et al. 2008, Trainer et al. 2008). They are the most diverse group of algae, totalling about 200000 species (Holtermann et al. 2010). They are structurally appealing because their cell walls, the frustules, are composed of amorphous silica. The morphology of the frustules (symmetry, shape, length and valve ornamentation) is used to support taxonomic classification and species identifi- cation (Scala \& Bowler 2001, Chepurnov et al. 2008). The frustules are observed by light microscopy (Chepurnov et al. 2008). In cases of ambivalence, scanning electron microscopy (SEM) can be used to further fine-tune diatom classification and/or identification. Furthermore, cytological and reproductive characteristics can be used to corroborate the identification based on cell wall morphology. Identification of diatoms targets important objectives besides contributing to the construction of scientific knowledge. Misleading identification and delays in correct diatom identification are critical when food safety (e.g. the presence of Pseudo-nitzschia spp., Bates et 
al. 1989) or biotechnological and nanotechnological applications (e.g. selection of diatoms for aquaculture purposes) are concerned.

Novel microbial identification methods need to be fast, less expensive and reliable (Santos et al. 2010). Matrix-assisted laser desorption/ionisation time-of-flight mass spectrometry (MALDI-TOF MS) is a powerful technique fulfilling all of the aforementioned requisites. This technique has been proposed as an identification method for bacteria (Shah \& Gharbia 2010), filamentous fungi (Santos et al. 2010), yeasts (Santos et al. 2011) and viruses (Franco et al. 2010, McAlpin et al. 2010). The MALDI-TOF MS technique has been used previously for diatom characterisation through the analysis of lipids (Vieler et al. 2007), chlorophylls (Suzuki et al. 2009), silaffins (Sumper et al. 2007) and polyamines associated with silica (Sumper \& Lehmann 2006) but not with the aim of establishing molecular mass fingerprinting profiles.

For microbial identification by the MALDI-TOF MS technique, the sample is normally covered with a UV-absorbing organic compound, called the matrix, leading to a crystallised mixture. Then, the crystallised sample is placed in a vacuum system that is targeted and irradiated with pulsed light from a laser (e.g. $\mathrm{N}_{2}$ Nd:YAG or other). The charged matrix molecules and/or clusters transfer protons onto the sample molecules (e.g. peptide or proteins) in the expanding plume. The generated ions are accelerated into the TOF analyser, where ions are separated according to their 'time of flight', which is a function of the ratio of molecular mass to charge $(\mathrm{m} / \mathrm{z})$. The $\mathrm{m} / \mathrm{z}$ ratio is determined by measuring velocities from accelerating ions to defined kinetic energies after calibration of the instrument with molecules of known

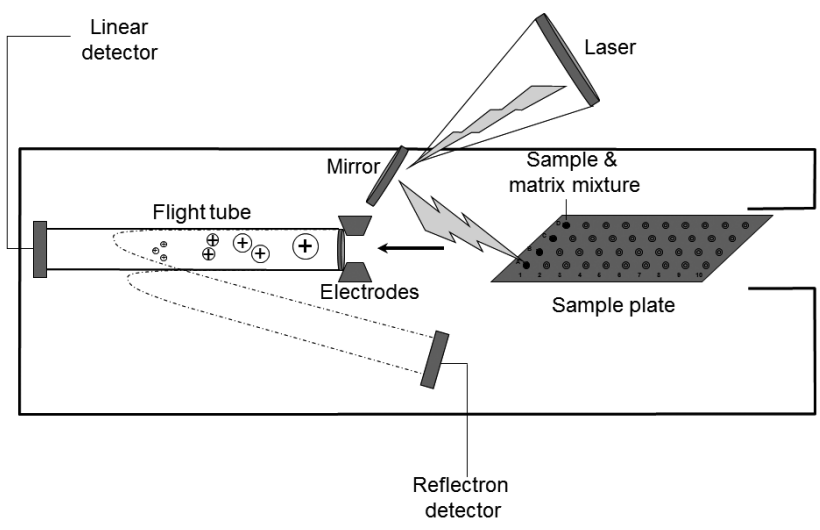

Fig. 1. Schematic representation of MALDI-TOF MS operation. After molecule ionisation, the separation of ions occurs inside the flight tube based on their molecular masses (Santos et al. 2010) molecular mass (Fig. 1, Passarini et al. 2013). The set of the $\mathrm{m} / \mathrm{z}$ ratio is displayed as a set of peaks, called the microorganism fingerprint. In MALDI-TOF MS, the linear mode set covers a huge mass range capable of providing the appropriate fingerprint for each kind of microorganism (Santos et al. 2010).

The aim of the present study was to develop a technique using MALDI-TOF MS as a high-throughput system for the rapid screening and characterisation of diatoms by investigating the influence of culture age in reproducibility of the resulting data.

\section{MATERIALS AND METHODS}

The diatoms used in this study included strains of Seminavis robusta, Coscinodiscus sp., Thalassiosira sp. and Cyclotella meneghiniana. For S. robusta, different strains and, within the same strain, different mating types were used $(84 \mathrm{~A}, 85 \mathrm{~A}, 85 \mathrm{As}$ and $85 \mathrm{Bs})$. Diatoms routinely grow through binary division, but a smaller half-frustule has to be built in each division, leading to a gradual size reduction, which ultimately would lead to programmed cell death. The recovery of cell size is made through sexual reproduction. $S$. robusta is one of the few diatoms with a heterothallic (dioecy) mating system, where two mating types exist (Chepurnov et al. 2002).

All of the diatoms were obtained from the Laboratory of Protistology and Aquatic Ecology culture collection (www.pae.ugent.be/collection.htm), University of Ghent, Belgium. Cyclotella meneghiniana was routinely maintained in Woods Hole modified Chu10 medium (Guillard \& Lorenzen 1972), and the other strains were routinely maintained in $f / 2$ medium (Guillard 1975). All cultures were synchronised and grown at room temperature, with a 12:12 h light:dark cycle at a photon flux density of $50 \mu \mathrm{mol}$ photons $\mathrm{m}^{-2}$ $\mathrm{s}^{-1}$. Possible contaminations and the health state of each culture were checked by directly observing the diatom cultures in the culturing flasks using an inverted optical microscope (Nikon Diaphot).

The experiments were designed to investigate reproducibility and hypothetical variation along culturing age. To establish a standardised protocol for the MALDI-TOF MS analysis for all diatom isolates, a first set of preliminary experiments was carried out only for the isolates Seminavis robusta 84A and Cyclotella meneghiniana. In this first set of preliminary assays, $S$. robusta $84 \mathrm{~A}$ and $C$. meneghiniana were grown for $40 \mathrm{~d}$ in 6 independent culturing flasks, and then each of these isolates was individually analysed by MALDI-TOF MS. 
Following the aforementioned experiments, a second set of 2 independent assays was conducted with Coscinodiscus sp., Thalassiosira sp., Seminavis robusta 84A and Cyclotella meneghiniana. For each diatom isolate, at least 2 individual samples were taken for the MALDI-TOF MS analysis.

For the MALDI-TOF MS analysis, a general protocol was established for all diatom isolates evaluated in the present study. The number of diatom cells varied according to the strain under study. Each diatom suspension in the liquid culture medium was centrifuged at $2000 \times g$ for $10 \mathrm{~min}$ to generate pellets containing ca. 200000 cells for Seminavis and Cyclotella isolates, ca. 40000 cells for Coscinodiscus isolates and ca. 4000 cells for Thalassiosira isolates.

The diatom pellets were then transferred into an aliquot containing $30 \mu \mathrm{l}$ of an acetonitrile aqueous solution, resulting in a green suspension, and $0.5 \mu \mathrm{l}$ was placed on the MALDI sample plate. When the liquid phase was almost evaporated, $0.5 \mu l$ of 2.5dihydroxybenzoic acid (DHB) matrix solution (75 mg $\mathrm{ml}^{-1}$ DHB in ethanol/water/acetonitrile [1:1:1; v/v/v] with $0.03 \%$ trifluoroacetic acid) was added and mixed gently. Finally, all samples were air-dried at room temperature and analysed in quadruplicate. Only spectra with at least 60 peaks were selected for further analysis to ensure that a correct ionisation of the sample took place. To ensure that no peaks were obtained from the culture medium ingredients, control experiments with cell-free culture medium were carried out.

The MALDI-TOF MS analysis was performed with a MALDI-TOF AXIMA-LNR system (Kratos Analytical). The instrument was equipped with a nitrogen laser $(337 \mathrm{~nm})$, where laser intensity was set above the threshold for ion production. The mass, ranging from 2 to $20 \mathrm{kDa}$, was recorded using the linear mode, with a delay of $104 \mathrm{~ns}$ and an acceleration voltage of $+20 \mathrm{kV}$. Final spectra were generated by summing 20 laser shots accumulated per profile and 50 profiles produced per sample, leading to 1000 laser shots per summed spectrum. The resulting peak lists were exported to the Spectral Archiving and Microbial Identification System (SARAMISTM) software package (AnagnosTec, www.anagnostec.eu), with which the final identification was obtained. The similarity between individual spectra is expressed as the relative or absolute number of matching mass signals after subjecting the data to a single-link agglomerative clustering algorithm. Detailed information about spectral clustering in SARAMIS ${ }^{\text {TM }}$ can be found in Pereira et al. (2014).

\section{RESULTS AND DISCUSSION}

As mentioned above, different authors have used MALDI-TOF MS as a tool to identify diatoms through the analysis of their lipids (Vieler et al. 2007), chlorophylls (Suzuki et al. 2009), silaffins (Sumper et al. 2007) or polyamines associated with silica (Sumper \& Lehmann 2006). These works were based on the analysis of these specific classes of compounds, with a mass range between 100 and $3000 \mathrm{Da}$, which is in accordance with the molecular weight of these molecular species extracted from diatoms cells. However, the genetic and, mainly, the proteomic information are not available in the literature for the diatoms species studied in this work. For this, a mass range from 2 to $20 \mathrm{KDa}$ was chosen, taking into consideration the proteomic information available for bacteria (Shah \& Gharbia 2010), filamentous fungi (Santos et al. 2010) and yeasts (Santos et al. 2011). For this kind of microbial cell, the molecular mass range between 2 and $20 \mathrm{kDa}$ corresponds to reproducible, highly abundant and constantly expressed biomarkers, such as ribosomal proteins (Santos et al. 2010, 2011, Shah \& Gharbia 2010).

Disruption of the cells was checked microscopically, so it is expected that intracellular molecules were analyzed by the present technique.

By conducting MALDI-TOF analyses between 5 and $40 \mathrm{~d}$, we could observe that each evaluated diatom species presented a specific age when the mass spectrum became reproducible. Below that limit, significant differences exist in the spectra obtained from different cultures, even at the same age and growth conditions. For all Seminavis robusta and Cyclotella meneghiniana isolates, the spectra were reproducible from the ninth day of culture (Fig. 2a), whereas for all Thalassiosira sp. and Coscinodiscus sp. isolates, the spectra were reproducible from the thirteenth day of culture (Fig. 2b).

The biological material was tested over a period of $18 \mathrm{~d}$ from the established minimum age limit, and the spectra were compared over time. Fig. 2c shows the MALDI-TOF spectra of Coscinodiscus sp. at 3 time intervals $(13,18$ and $30 \mathrm{~d}$ of culture). In these spectra, there is evidence that the mass spectra change with diatom age, which in turn indicates the existence of different compounds over time. The evaluated compounds are within a mass range from 2 to $20 \mathrm{KDa}$ and probably are not ribosomal proteins, since these should not vary so significantly with age. But it is of utmost importance to recognize that these spectra, although varying with time, were reproducible as long as the minimum age limit was respected: at least 

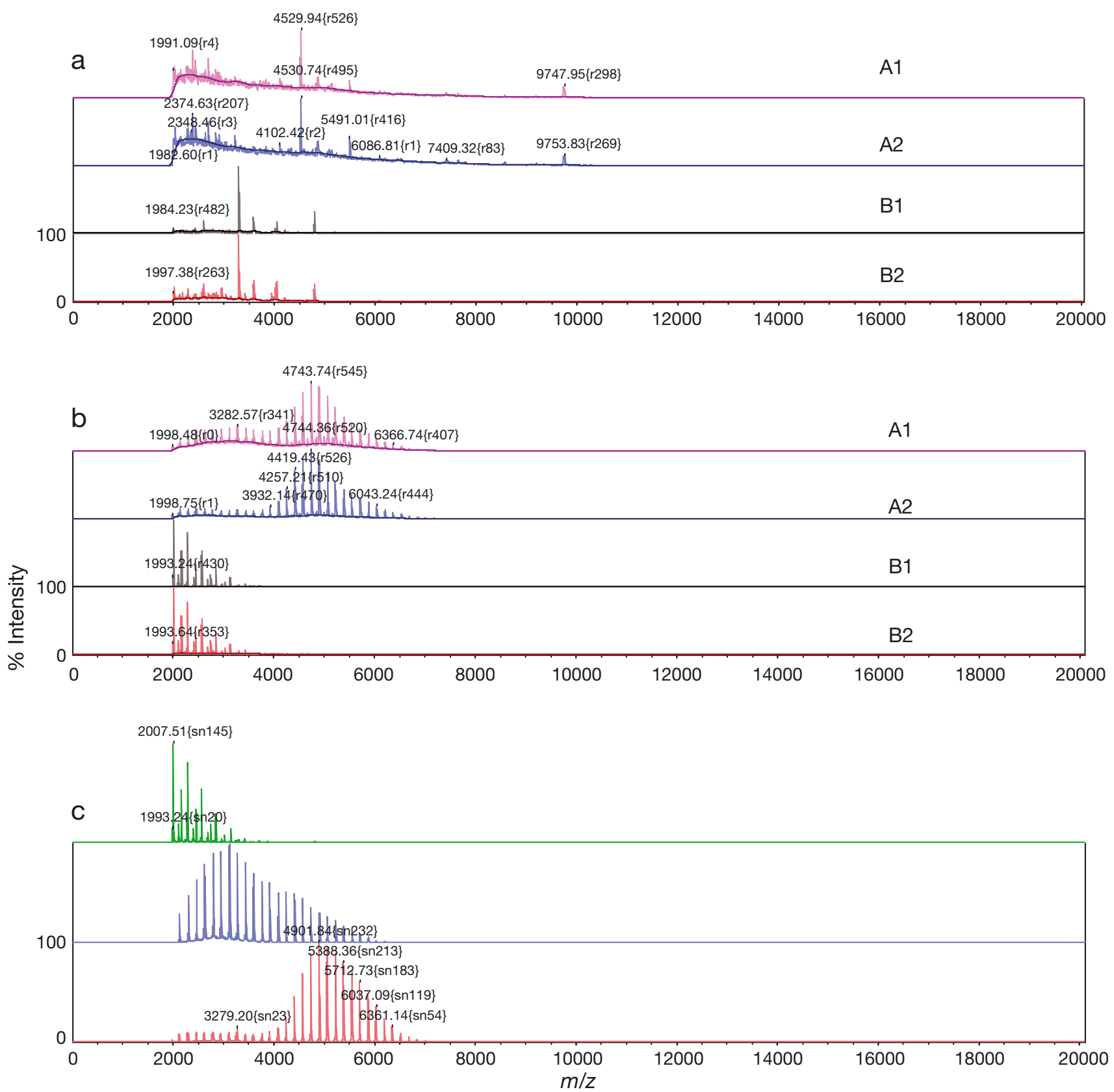

Fig. 2. Reproducible MALDI-TOF MS mass spectra for (a) Cyclotella meneghiniana (A1, A2) and Seminavis robusta (B1, B2) at $9 \mathrm{~d}$ old and (b) Thalassiosira sp. (A1, A2) and Coscinodiscus sp. (B1, B2) at $13 \mathrm{~d}$ old and (c) the same strain of Coscinodiscus sp. when prepared and analyzed at 3 different time intervals (13, 18 and $30 \mathrm{~d}$ old $)$

2 culture flasks were used to generate 8 MALDI-TOF MS spectra for each of the studied species, where at least 1 flask generated 4 MALDI-TOF MS spectra.

Using the reproducible spectral data of the second set of experiments, a cluster analysis of the MALDITOF MS mass spectral data was performed. This statistical analysis based on mass spectral signatures allowed the grouping of all isolates into clusters according to their species designation. The dendro- gram presented in Fig. 3 shows that the MALDI-TOF MS is able to separate the different genera of diatoms analysed.

The first clade of the dendogram, at a threshold of about $30 \%$ similarity, is between the pair including the freshwater Cyclotella meneghiniana and the marine Thalassiosira sp. (both centric) and the pair including the pennate Seminavis robusta and the centric Coscinodiscus sp. (both marine). C. meneghi- 
$\begin{array}{llllllllllll}0 & 10 & 20 & 30 & 40 & 50 & 60 & 70 & 80 & 90 & 100 & \text { Sample }\end{array}$
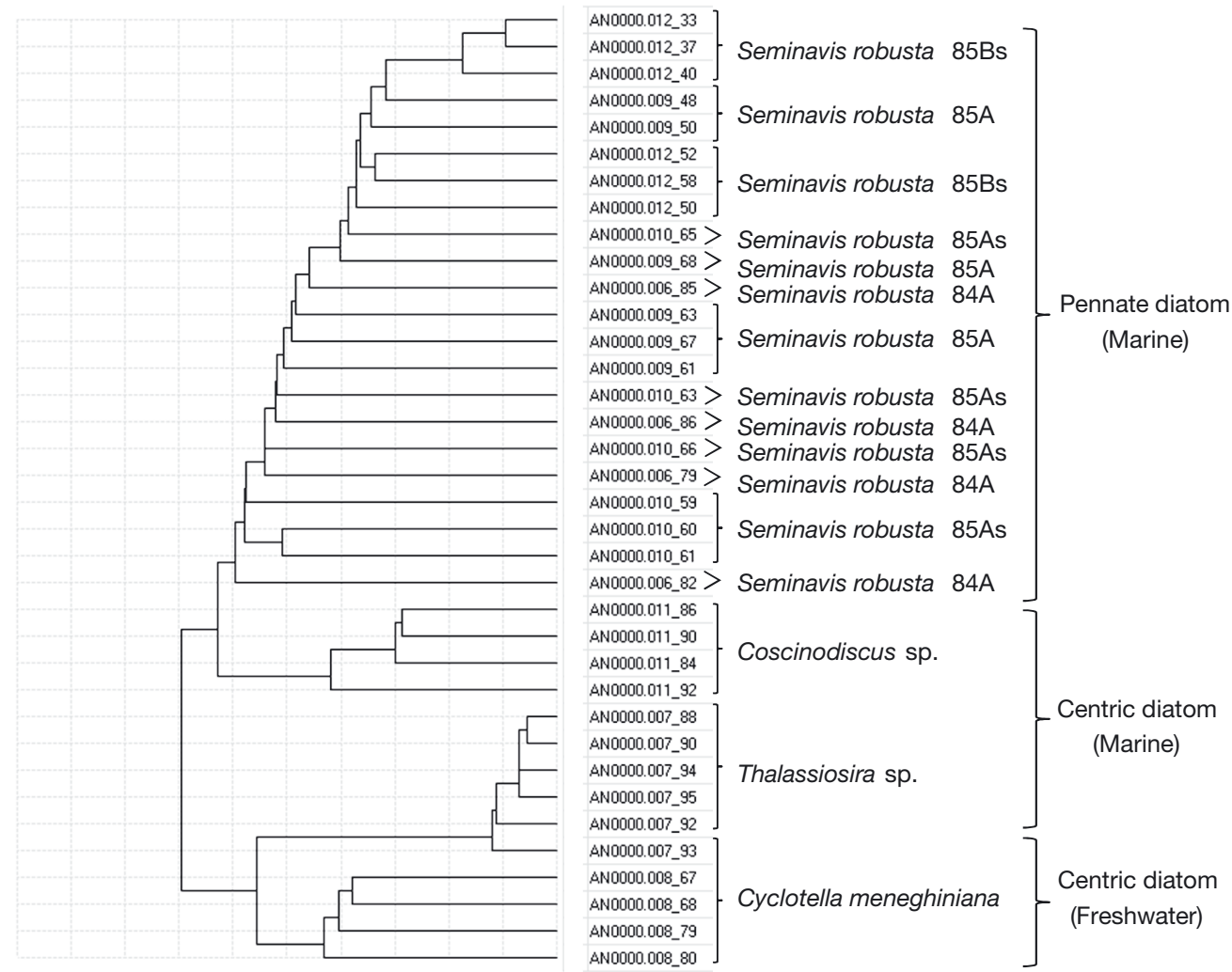

Fig. 3. MALDI-TOF MS spectra-based dendrogram of the diatoms studied in this work using cultures older than $9 \mathrm{~d}$ for Seminavis robusta strains and Cyclotella meneghiniana, and $13 \mathrm{~d}$ for Thalassiosia sp. and Coscinodiscus sp.

niana and Thalassiosira sp. belong to the order Thalassiosirales, so a close relation between their spectra was expected. This was confirmed, and the two diatom species were only distinct at a threshold of about $44 \%$. Additionally, the pair S. robusta/Coscinodiscus sp. could be identified at a similarity threshold of $38 \%$.

Seminavis robusta corresponds to the group of samples that has the lowest similarity among them when compared to the other diatoms (Coscinodiscus sp., Thalassiosira sp. and Cyclotella meneghiniana), which is in accordance with the fact that Seminavis robusta samples come from cultures of different strains and, even when belonging to the same strain, belong to different mating types. On the other hand, $S$. robusta strains $84 \mathrm{~A}, 85 \mathrm{~A}$, $85 \mathrm{As}$ and $85 \mathrm{Bs}$ grouped together without a clear spectral differentiation for each strain or mating type. The analysed $S$. robusta cultures belonged to different mating types; $S$. robusta $84 \mathrm{~A}$ and 85Bs belonged to mating type 1 , and $S$. robusta 85As and $85 \mathrm{~A}$ belonged to mating type 2 (Chepurnov et al. 2008).

\section{CONCLUSIONS}

When the culturing age is known, MALDI-TOF MS appears to be a powerful high-throughput mass spectra method to discriminate diatom genera. The MALDI-TOF MS equipment is straightforward to operate and cost-effective on an individual sample basis. On the other hand, all studied diatoms presented a specific age when the mass spectra became reproducible. The culturing age limit for diatom identification by MALDI-TOF MS was $9 \mathrm{~d}$ old for Seminavis robusta and Cyclotella meneghiniana and $13 \mathrm{~d}$ old for Thalassiosia sp. and Coscinodiscus sp. However, reproducibility was not accompanied by the compound's identity over time. Impacts can be significant if the MALDI-TOF MS technique is used to identify specific diatoms, as spectra of different culturing ages are not comparable even if the age limit when they are reproducible is surpassed. Below that age limit, they are not reproducible, so different spectra are expected from different cultures or assemblages even with similar age and growth conditions. Furthermore, the distinctive molecular mark- 
ers are located in a region encompassing compounds of a molecular weight between 2000 and 6000 . They come up at a defined moment of the physiological state, meaning they are not structural compounds. Regardless, as they are substantially different in each strain, they might be useful as molecular fingerprints. Further studies involving their characterisation will help to associate these molecules with specific physiological states.

The present results are fundamental to development of a comprehensive protocol on the use of the MALDI-TOF MS technique to differentiate or identify diatoms. Many challenges have now arisen from the present work, including the possibility of using the MALDI-TOF MS technique to discriminate diatoms to the species level, to identify which types of compounds are being reflected in the present spectra and to determine how they vary with culturing age.

Acknowledgements. The authors sincerely acknowledge W. Vyverman and P. Vanormelingen for kindly providing the diatom cultures. The authors thank the FCT Strategic Project PEst-OE/EQB/LA0023/2013, the FCT Project RECI/ BBB-EBI/0179/2012 (FCOMP-01-0124-FEDER-027462) and the Project 'BioENV - Biotechnology and Bioengineering for a sustainable word', REF. NORTE-07-0124-FEDER-000048, co-funded by the Programa Operacional Regional do Norte (ON.2 - O Novo Norte), QREN, FEDER.

\section{LITERATURE CITED}

Bates SS, Bird CJ, de Freitas ASW, Foxall R and others (1989) Pennate diatom Nitzschia pungens as the primary source of domoic acid, a toxin in shellfish from eastern Prince Edward Island, Canada. Can J Fish Aquat Sci 46: 1203-1215

Chepurnov VA, Mann DG, Vyverman W, Sabbe K, Danielidis DB (2002) Sexual reproduction, mating system and protoplast dynamics of Seminavis (Bacillariophyceae). J Phycol 38:1004-1019

Chepurnov VA, Mann DG, Dassow P, Vanormelingen P, Gillard J, Inzé D, Vyverman W (2008) In search of new tractable diatoms for experimental biology. Bioessays 30: 692-702

Franco CF, Mellado MCM, Alves PM, Coelho AV (2010) Monitoring virus-like particle and viral protein production by intact cell MALDI-TOF mass spectrometry. Talanta 80:1561-1568

Guillard RRL, Lorenzen CJ (1972) Yellow-green algae with chlorophyllide $C$. J Phycol 8:10-14

Guillard RRL (1975) Culture of phytoplankton for feeding marine invertebrates. In Smith WL, Chanley $\mathrm{MH}$ (eds)

Editorial responsibility: Christine Paetzold, Oldendorf/Luhe, Germany
Culture of marine invertebrate animals. Plenum Press, New York, NY, p 26-60

Gordon R, Losic D, Tiffany MA, Nagy SS, Sterrenburg FAS (2009) The glass menagerie: diatoms for novel applications in nanotechnology. Trends Biotechnol 27:116-127

Gröger C, Sumper M, Brunner E (2008) Silicon uptake and metabolism of the marine diatom Thalassiosira pseudonana: solid-state ${ }^{29} \mathrm{Si}$ NMR and fluorescence microscopic studies. J Struct Biol 161:55-63

> Holtermann KE, Bates SS, Trainer VL, Odell A, Armbrust EV (2010) Mass sexual reproduction in the toxigenic diatoms Pseudo-nitzschia australis and $P$. pungens (Bacillariophyceae). J Phycol 46:41-52

McAlpin CR, Cox CR, Matyi SA, Voorhees KJ (2010) Enhanced matrix-assisted laser desorption/ionization timeof-flight mass spectrometric analysis of bacteriophage major capsid proteins with $\beta$-mercaptoethanol pretreatment. Rapid Commun Mass Spectrom 24:11-14

Passarini MRZ, Santos C, Lima N, Berlinck RGS, Sette LD (2013) Filamentous fungi from the Atlantic marine sponge Dragmacidon reticulatum. Arch Microbiol 195: 99-111

Pereira L, Dias N, Santos C, Lima N (2014) The use of MALDI-TOF ICMS as an alternative tool for Trichophyton rubrum identification and typing. Enferm Infecc Microbiol Clin 32:11-17

> Santos C, Paterson RMR, Venâncio A, Lima N (2010) Filamentous fungal characterisations by matrix-assisted laser desorption/ionisation time of flight mass spectrometry. J Appl Microbiol 108:375-385

Santos C, Lima N, Sampaio P, Pais C (2011) Matrix-assisted laser desorption/ionization time-of-flight intact cell mass spectrometry (MALDI-TOF-ICMS) to detect emerging pathogenic Candida species. Diagn Microbiol Infect Dis 71:304-308

- Scala S, Bowler C (2001) Molecular insights into the novel aspects of diatom biology. Cell Mol Life Sci 58:1666-1673

Shah HN, Gharbia SE (eds) (2010) Mass spectrometry for microbial proteomics. Wiley, Chichester

> Sumper M, Lehmann G (2006) Silica pattern formation in diatoms: species-specific polyamine biosynthesis. ChemBioChem 7:1419-1427

Sumper M, Hett R, Lehmann G, Wenzl S (2007) A code for lysine modifications of a silica biomineralizing silaffin protein. Angew Chem 46:8405-8408

- Suzuki T, Midonoya H, Shioi Y (2009) Analysis of chlorophylls and their derivatives by matrix-assisted laser desorption/ionization-time-of-flight mass spectrometry. Anal Biochem 390:57-62

Trainer VL, Hickey BM, Bates SS (2008) Toxic diatoms. In: Walsh P, Smith S, Fleming L, Solo-Gabriele H, Gerwick $\mathrm{W}$ (eds) Oceans and human health: risks and remedies from sea. Elsevier, New York, NY, p 219-221

Vieler A, Wilhelm C, Goss R, Süß R, Schiller J (2007) The lipid composition of the unicellular green alga Chlamydomonas reinhardtii and the diatom Cyclotella meneghiniana investigated by MALDI-TOF MS and TLC. Chem Phys Lipids 150:143-155

Submitted: December 6, 2012; Accepted: November 11, 2013 Proofs received from author(s): February 6, 2014 